\title{
Blind Painful Eye Revealing Spontaneously Regressed Retinoblastoma in Young Patient
}

\author{
Hadijah $^{1}$, Halimah Pagarra ${ }^{1}$, Suliati P.Amir ${ }^{1}$, Andi Pratiwi ${ }^{1}$ \\ ${ }^{1}$ Department of Ophthalmology, Faculty of Medicine, Hasanuddin University \\ Wahidin Sudirohusodo Hospital, Makassar \\ E-mail: hadijah.djoko@gmail.com
}

\begin{abstract}
Purpose: Spontaneous regression is a rare but well-known feature of retinoblastoma, which is predicted in approximately 2 of every 100 cases. Unilateral, unifocal spontaneous regression of retinoblastoma is infrequent; bilateral multifocal spontaneous regression of retinoblastoma is even more extraordinary. In many of the previously reported cases, the eye (or eyes) containing the spontaneously regressed tumor has been phthisical and blind. Herein, we describe the case of a young woman with unilateral, spontaneously regressed retinoblastoma (RB), with inflamed, blind, and phthisis in the left eye as an uncommon presentation of spontaneously regressed retinoblastoma

Methods: This is a case report: An 18-year-old woman came to the outpatient clinic with a chief complaint of pain in the left eye since 1 month prior and a history of dysfunctional "cat's" eye since childhood.

Results: Exotropia and atrophy bulbi was noticed in the left eye. Anterior segment examination revealed signs of inflammation including conjunctival hyperemia, shallow anterior chamber, rubeosis iridis, and posterior synechia with white "chalky" mass suggestive calcification. Bscan ultrasonography showed a point like lesion and solitary solid intraocular mass with calcification in vitreus. Orbital computed tomography scan (CT-Scan) showed atrophy of the left eye with intraocular calcification without optic nerve and extraocular muscle involvement. Enucleation and histopathology examination were done and revealed undifferentiated retinoblastoma beyond the sign of metastasis in other parts of the eye.

\section{Conclusion}

Blind painful eye may present as the main feature of spontaneously regressed retinoblastoma that possibly caused by an inflammatory reaction.
\end{abstract}

\section{Keywords}

Retinoblastoma, Spontaneous regression, Inflammation.

\section{INTRODUCTION}

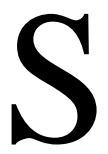
pontaneous regression of retinoblastoma is a well-known phenomenon that is said to occur more frequently in retinoblastoma than in any other malignant neoplasm, which is a rare but well-known feature of retinoblastoma, and predicted in approximately 2 of every 100 cases. Clinical diagnosis of spontaneous regression can be made with relative certainty only in patients having viable retinoblastoma in the same or the opposite eye or in patients with a pathologically confirmed family history of retinoblastoma. Unilateral, unifocal spontaneous regression of retinoblastoma is infrequent; bilateral multifocal spontaneous regression of retinoblastoma is even extraordinary. In many of the previously reported cases, the 
eye (or eyes) containing the spontaneously regressed tumor has been phthisical and blind. ${ }^{1,2}$

In this paper, we aimed to report a rare case of a spontaneously regressed retinoblastoma (RB) which was detected in young adult (18-year old) patient, presented with inflamed, blind, and phthisis in the left eye.

\section{CASE DESCRIPTION}

A 23 An eighteen-year-old woman was referred to the outpatient clinic with chief complaint of a painful eye since 1 a month prior associated with history of blind "cat's eye" and exotropia since childhood. A family history of similar complain and prematurity was inattentive. There was a history of spontaneous hyphema. Visual acuity was 20/20 and no light perception in the right and left eye, respectively. Slitlamp examination in the left eye revealed inflammation including conjunctival hyperemia, shallow anterior chamber, rubeosis iridis, posterior synechia, and white "chalky" mass attached to the inferior iris, suggestive calcification (Fig. 1). No signs of enlarged lymph nodes in the preauricular, submandibular, jugular, axial and inguinal and laboratory examination were normal.

In the vitreus cavity, B-scan ultrasonography showed a point like lesion and solitary solid intraocular mass with calcification (Fig.2). Orbital CT-Scan of the left eye displayed bulbar atrophy with intraocular calcification. No orbital abnormalities were seen on the CTscan. (Fig.3).

It was difficult to differentiate regressed retinoblastoma from active recurrent retinoblastoma, due to posterior synechia and white mass precluded ophthalmoscopic visualization, even though inflammation and pain can be a warning sign of the recurrence RB. Overall, most commonly tumor regrowth appeared in the eyes of children diagnosed at a very young age or those with family history of
$\mathrm{RB}^{3}$ which is absent in the current case. We performed enucleation followed by histopathology examination to determine the diagnosis, which clarified the tissue as undifferentiated retinoblastoma, without rosettes and confirmed there was no sign of cornea, ciliary body and optic nerve attachment with the tumor (Fig 4,5). Therefore, chemotherapy was not suggested.

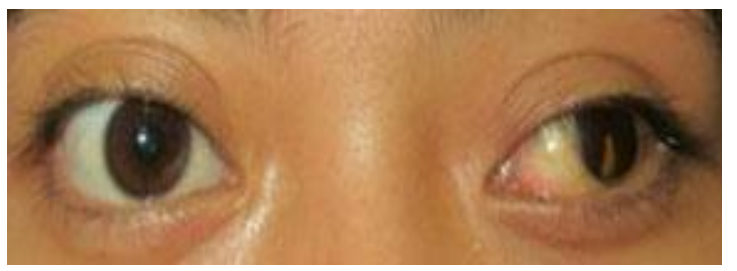

A

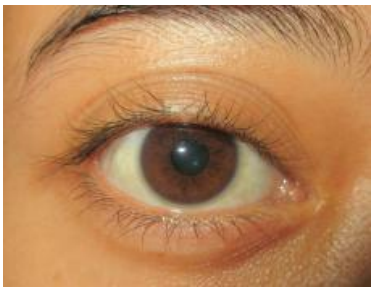

B

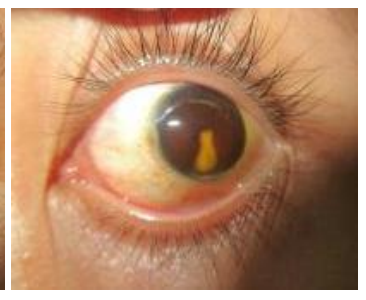

C
Fig 1. A. Mild microphthalmia of the left eye along with exotropia in primary position. B. The right eye was normal. C. Inflammation in the left eye showed conjunctival hyperemia, shallow anterior chamber, rubeosis iridis, posterior synechia, and white mass lession suggestive calcification.
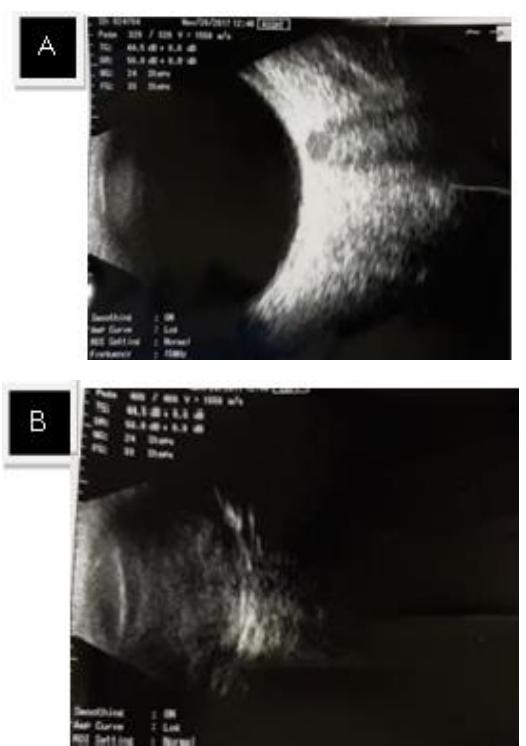

Fig 2. A. Normal USG in right eye. B. USG in left eye showed point like lesion and solitary 
mass like lession, suggest calcification in vitreus.

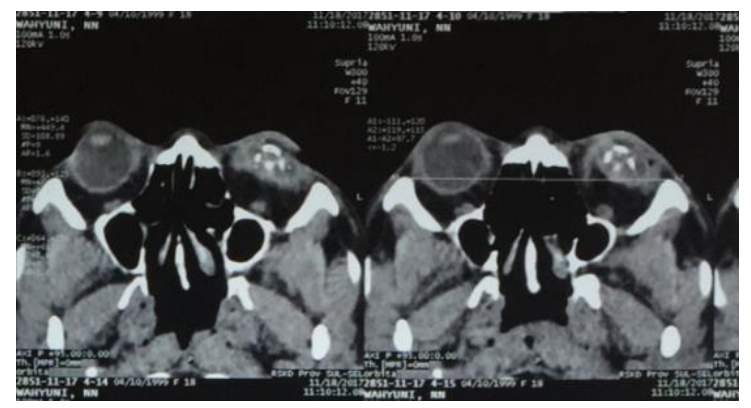

A

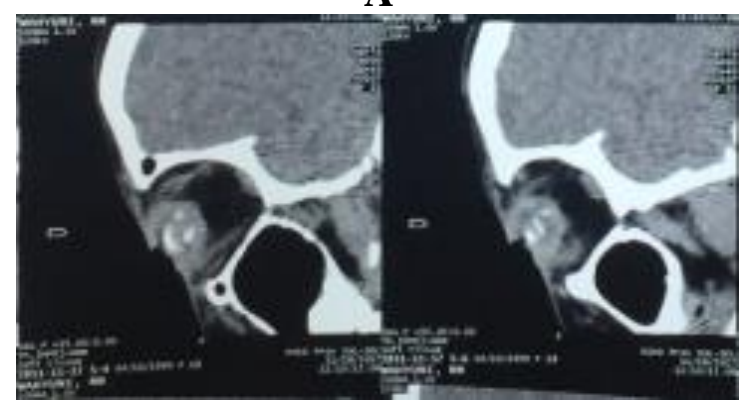

B

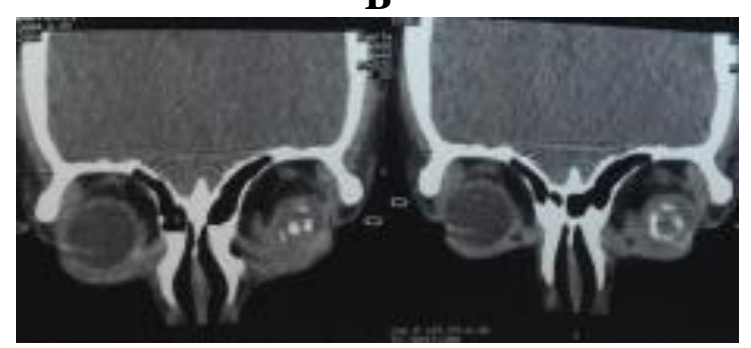

C

Fig 3. Orbital CT-Scan A. Axial, B. Sagittal, C. Coronal, demostrated left bulbar atrophy with intraocular calcification, suggestive retinoblastoma, with normal optic nerve and extraocular muscles.

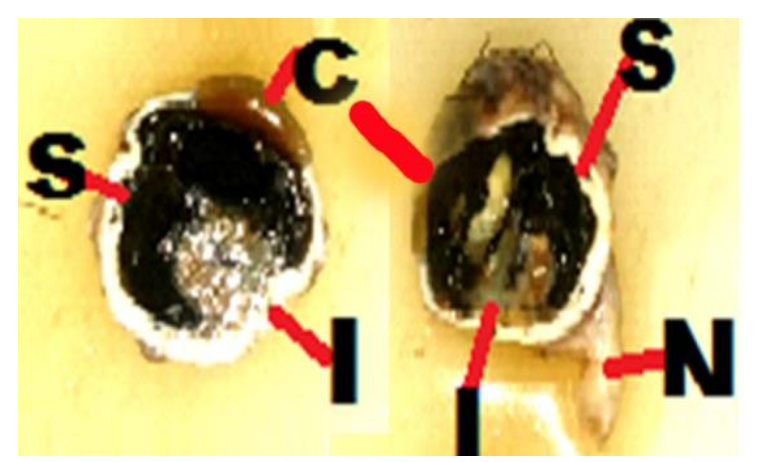

Fig 4. Macroscopic view of the left eye after enucleated. White mass in vitreous cavity (arrow). $\mathrm{C}=$ cornea, $\mathrm{S}=$ superior, $\mathrm{I}=$ inferior, $\mathrm{N}=$ optic nerve

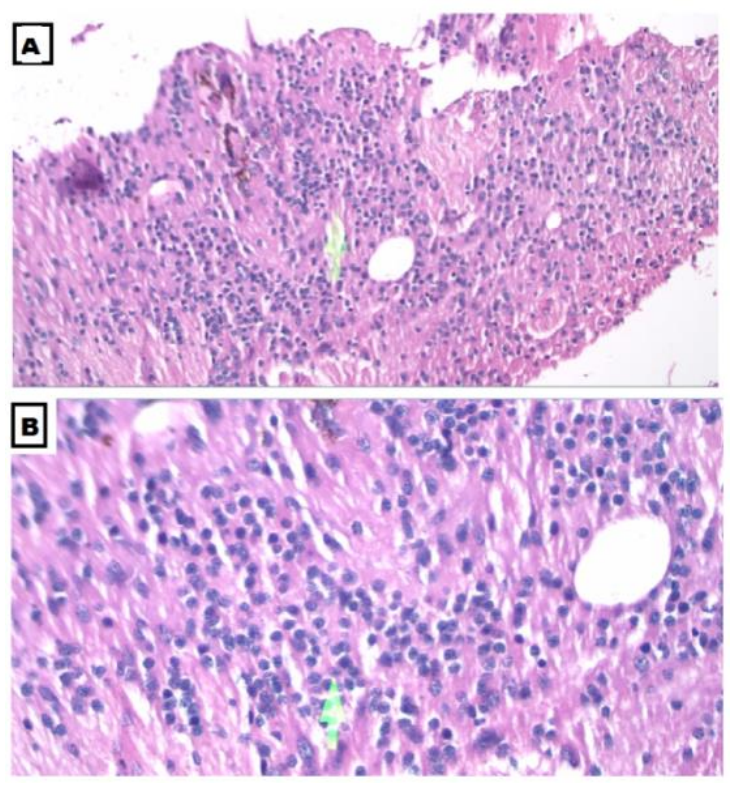

Fig 5. A.Histopathology (Hematoxylin and Eosin, x20). B. (Hematoxylin and Eosin, x40), displayed small undifferentiated cells with hyperchromatic nuclei and scanty cytoplasm without rosettes form

A lot of well-documented cases of spontaneously regressed retinoblastoma have been published in the literature. A number of unilateral cases have been diagnosed based on histopathological results in patients with atrophy bulbi of unknown causes. ${ }^{2}$ In most of the reported cases, the eye (or eyes) having the spontaneously regressed tumor has been atrophy and blind. These features consistent with our patient who was presented with atrophy bulbi and blind. In addition, our patient had a history of leukokoria and exotropia since childhood which are classical findings for retinoblastoma. More unusual indicators include neovascular glaucoma and spontaneous hyphema ${ }^{4}$ were manifested in this case. Spontaneous hyphema has been reported as a remarkable sign in an advance case which presumably originates from rubeosis iridis and considered as atypical presentations of retinoblastoma. Rubeosis iridis is a hallmark of advanced tumor and considered a poor prognosis for globe salvage and vision; thus, enucleation is typically advised. ${ }^{5}$ Moreover, these appearances associated with a higher incidence of high 
risk histopathology conclusions as reported by Shields and Kayshap et al. ${ }^{6}$

\section{DISCUSSION}

There are some theories about the pathogenesis of spontaneous regression RB. The most popular one is the vascular theory, stated that regression arises due to insufficient vascular in the fast-growing tumor, outgrows its blood supply, becomes necrotic, and finally regresses. Another theory postulates that an immunological process may take responsibility for the regression. Immunological complex deposits found in several retinoblastomas that have undergone spontaneous necrosis, along with elevated values of circulating immune complexes. The inflammatory reaction is one of the characteristics of regressed RB. ${ }^{1}$ Constantly, our patients came up with inflamed eye, and posterior synechia, which are quite suspicious to be the signs of immunological reactions that flared-up after regression takes place and assumed to be the cause of the pain. Another case of spontaneous RB with inflammation and phthisis bulbi previously reported by M.Padma et al in $2015,{ }^{1}$ In fact, ocular inflammation is unusual in RB. Shields et al in 1991 reported 5 cases of ocular inflammation in RB, whereas most of their patient with necrotic RB confined within the eye came without high fever and leucocytosis but in general presented with marked leukocoria and calcified tumor mass in CT-Scan. ${ }^{4}$ This report is similar with the present case in which the laboratory value was normal, but signs of inflammation is notable.

Regressed retinal tumors, bilateral or unilateral, mostly reported with retained useful vision because the lesions were in outer macular area. ${ }^{2}$ However, our patient had impaired visual acuity probably due to the location of the tumor was surrounding the macula, as seen in the macroscopic figure (Fig. 4).

Ultrasound B-scan is the primary and most important study to confirm the presence of an intraocular mass arising from the retina, to measure the dimensions of mass, to detect associated vitreous seeds or retinal detachment, and to exclude optic nerve invasion. This examination exhibits a dome-shaped solid mass growth endophyticly within the vitreous cavity, with point-like lesion represent calcification. Further radiologic imaging was CT-scan, considered to be the gold standard imaging modality to detect intraocular calcifications. $\quad{ }^{[7,8,9]} \mathrm{A}$ retrospective study of 21 cases of retinoblastoma using high-resolution $\mathrm{CT}$ found intraocular calcification in $83 \%$ of the cases. Based on CT-Scan of the orbit showed atrophy bulbi in the left eye with intraocular calcification, highly suggestive of retinoblastoma. It is known that calcium inhibits tumor growth, and Reese has suggested that the calcium in cases of spontaneous regression has been sufficiently elevated to inhibit growth. Calcium deposits represent the necrotic areas in all cases of retinoblastoma. ${ }^{10}$ Most of previously reported cases of spontaneous regressed RB were diagnosed based on one or more of the subsequent three criteria: (1) Characteristic fundus picture, (2) Clinical diagnosis bilateral RB with the removal of the most affected eye, and in most cases microscopic verification, followed by regression in the fellow eye, (3) The presence of calcified tumor in a removed phthisical eye. ${ }^{10}$ Although our patient did not follow all the three criteria, enhanced radiologic tests proved the area of calcification as the sign of necrosis process within the intraocular tumor.

This patient underwent enucleation and macroscopic anatomy showed an intraocular mass on the inferior side within the vitreous cavity. The tumor had endophytic growth. Endophytic growth occurs when the tumor breaks through the internal limiting membrane and has an ophthalmic appearance of a white-to-cream mass showing either no surface vessels or small irregular tumor vessels. ${ }^{11}$

The typical histopathological findings in spontaneously regressed retinoblastoma consist of calcified tumor 
nest and necrosis of the surrounding retina. ${ }^{12}$ Histopathology features were recorded regarding growth pattern, tumor differentiation, and invasion into the anterior chamber, iris, ciliary body, choroid, sclera, orbit, or optic nerve. ${ }^{13}$ Histopathology result in the present case was atypical for spontaneous regressed $\mathrm{RB}$, but undifferentiated retinoblastoma, without the involvement of any other part of the eye, include the cornea, corpus ciliar, optic nerve, and no rosettes were identified. Undifferentiated retinoblastoma is composed of small, round, densely packed cells with hyperchromatic nuclei and scant cytoplasm. Several degrees of photosensory differentiation have been described and are characterized by distinctive arrangements of tumor cells: the Homer-Wright rosettes, most often seen in other neuroblastic tumors such as neuroblastoma and medulloblastoma and infrequently seen in retinoblastoma; the Flexner- Wintersteiner rosettes, specific for retinoblastoma and seen in $70 \%$ of the tumors; and the fleurettes, structures more characteristic of well-differentiated tumors. ${ }^{14,15}$

It is sometimes difficult to distinguish RB and other congenital ocular anomalies. Intraocular calcification, which is considerably important in RB patient was found in the present case; nevertheless, this sign is not a pathognomonic diagnostic feature in retinoblastoma as it can also show up in other anomalies e.g Persistent Fetal Vasculature (PFV) and Coat's disease. Hence, calcification has rarely been reported in adult $\mathrm{RB}$, and this stresses that our case is unlikely to be an adult form of RB and more likely to be regressed type. The other differential diagnosis is Retinoma/Retinocytoma, a benign tumor of the retina that results from a partially differentiated retinoblast. As described by Gallei and associates, retinoma mimics retinoblastoma clinically, and carries same genetic risk and risk to fellow eye, calcification, but without mitosis and necrosis. ${ }^{1}$ After all, an active RB remains to be a possible differential diagnosis in this patient, nonetheless, in ophthalmoscope examination, an active RB appears as a flat, transparent slightly white lesion in the sensory retina while tumor in bigger size appears as solid white with dilated tortuous retinal vasculature that feeds and drain the tumor. Unfortunately, this examination was not performed in the current case since the posterior synechia and the white mass that adjacent to the surrounding pupil blocked the visual axis.

Management of retinoblastoma should be guided by the objectives save life, retain anatomical integrity of the eye, preserve vision, and obtain good cosmetic results. ${ }^{16}$ Conservative modalities that can result in globe salvage, and preservation of functional vision are being successfully employed in a significant number of patients, enucleation remains the standard of care for advanced intraocular tumors. ${ }^{17}$ Our patient underwent enucleation surgery. Enucleation is indicated for unilateral retinoblastomas that fill most of the globe and when there is little hope of salvaging vision. Enucleation was the primary treatment modality in the majority of reported adult cases of retinoblastoma, as the lesions were detected at a fairly advanced stage and each patient had one normal unaffected eye. Few patients underwent external beam radiotherapy. ${ }^{16}$ Another treatment for retinoblastoma in adult are laser photocoagulation, local chemotherapy, Superselective intraarterial chemotherapy, and systemic chemotherapy. ${ }^{11,18}$

Systemic chemotherapy has been used to treat intraocular Retinoblastoma. The common indications of chemotherapy for retinoblastoma include tumors that are large and cannot be treated with local therapies alone, recurrent and relapsed tumors, and as an adjuvant therapy to enucleation in cases of highrisk histopathologic characteristics. The most commonly used chemotherapeutic agents include carboplatin vincristine and etoposide. Chemotherapy is used to reduce the size of the tumor to allow local 
therapies. ${ }^{17}$ However, our patient was not suggested to be managed with chemotherapy for the reason that the tumor did not extend to other parts of the eye and systemically. As mentioned above, no signs of lymphatic involvement were detected.

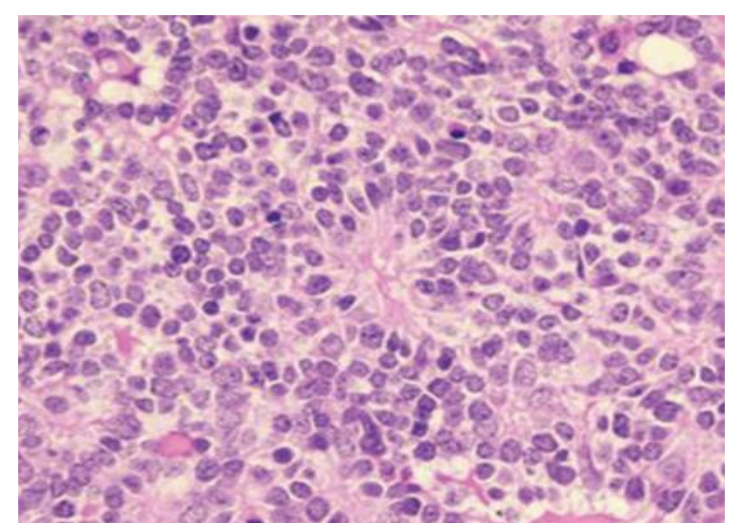

Fig 6. Well-differentiated retinoblastoma, displaying Homer-Wright rosettes and FlexnerWintersteiner rosettes (Hematoxylin and Eosin, $\times 200$ ).

\section{CONCLUSION}

Spontaneous regression of retinoblastoma may present with varying signs and symptoms, including inflammation and blind painful eye. Possibilities of other intraocular tumors and congenital anomaly, as well as active retinoblastoma, are considerable differential diagnoses. Enucleation followed by histopathology examination is essential to determine the diagnosis of retinoblastoma.

\section{REFERENCES}

1. Murray AD. An Approach to Some Padma M, Shivanand N, Vishwanath K. Spontaneous regression of retinoblastoma-A Case Report. MRIMS Journal of Health Sciences 2015; 3(3).

2. Sanborn GE, Augsburger JJ, Shields JA. Spontaneous regression of bilateral retinoblastoma. British Journal of Ophthalmology 1982; 66:685-690.

3. Gelaw Y, Shoukry SM, Othman IS. Unusually very late0onset new growth of intraocular retinoblastoma:A case report and review of literature. American Journal of Ophthalmology Case reports $2017 ; 5$ : 130-133.

4. Shields JA, Shields C, Suvarnamani C, et al. Retinoblastoma Manifesting as Orbital Cellulitis. American Journal of Ophthalmology 1991;112:442-449.

5. Kumar Singh S, Das D, Bhattacharjee H, Biswas J, Kuri G. A rare case of adult onset retinoblastoma. Oman Journal of Ophthalmology 2011;4;25-7

6. Chairibi F, Bhallil S, Benatiya I, et al. Hyphema Revealing Retinoblastoma In Childhood. A Case Report. Bull.Soc.belge Ophthalmol 2011; 318:41-43.

7. Garg V, Gupta A, Pruthi SK, Khare P. Retinoblastoma in an adult. J Cytol 2018;35:120-4.

8. James SH, Halliday WC, Branson HM. Best cases from the AFIP: Trilateral retinoblastoma. Radiographics 2010;30:833-7.

9. BeetsTan RG, Hendriks MJ, Ramos LM, Tan KE. Retinoblastoma: CT and MRI. Neuroradiology 1994;36:59-62.

10. Nehen JH. Spontaneous Regression of Retinoblastoma. Acta Ophthalmologica 1975;53:647-651.

11. Ellsworthl RM. The practical management of Retinoblastoma. Management of retinobalstoma 1969;67:462-534

12. Sengupta S, Pan U, Khetan V. Adult onset retinoblastoma. Indian $\mathrm{J}$ Ophthalmol 2016;64:485-91.

13. Zhang X, Zheng L, Gao F, Dong F. Adult onset retinoblastoma: A case report. Chin Med J (Engl) 2015;128:133-4.

14. Rodriguez C, Pappo AS, Holland-Frei Cancer Medicine, Retinoblastoma 2003

15. Preethi S. Retinoblastoma-Demographic Features, Clinical Presentations, Treatment Modalities and Outcomes - In a Tertiary Care Centre. MS Branch III Ophthalmology 2006;1-128

16. Sharifzadeh M, Ghassemi F, Amoli FA, Rahmanikhah E, Tabatabaie SZ. Retinoblastoma in adults: A case report and literature review. J Ophthalmic Vis Res 2014;9:388-91

17. Chawla B, Jain A, Azad R. Conservative treatment modalities in retinoblastoma. Indian J Ophthalmol 2013;61:479-85.

18. Shields CL, Shields JA. Diagnosis and management of retinoblastoma. Cancer Control 2004;11:317-27. 